\title{
Erratum: Symmetric Linear Collision Operators in Kinetic Theory ${ }^{1}$
}

\author{
E. G. D. Cohen and I. M. de Schepper
}

Received June 12, 1987

Omitted from the Acknowledgment was the support of the Department of Energy under contract DE-AC02-81ER10807.

\footnotetext{
${ }^{1}$ This paper appeared in J. Stat. Phys. 46:949-969 (1987).
} 